\title{
Eddy Current Linear Speed Sensor
}

\author{
Mehran Mirzaei ${ }^{1}$, Pavel Ripka ${ }^{1}$, Andrey Chirtsov ${ }^{1}$ and Jan Vyhnanek ${ }^{1}$ \\ ${ }^{1}$ Faculty of Electrical Engineering, Czech Technical University, Prague, Czech Republic
}

\begin{abstract}
Novel eddy current speed sensor with axisymmetric coils and solid iron rod as moving part is presented. The analysis is performed both for DC and AC coil currents and for variable iron rod translational speed. The coil inductance and induced voltage results using analytical method and finite element method calculation are compared with measured values. Two different coil configurations are used for simulations and measurement.
\end{abstract}

Index Terms-Analysis, eddy current, linear, speed sensor.

\section{INTRODUCTION}

$\mathrm{T}$ HE SPEED sensors are used for rotating and translational objects in many applications. Different types of sensors such as non-magnetic optical sensor or variable reluctance, eddy current based and Hall effect magnetic sensors could be utilized for speed sensing. Magnetic sensors principles are based on magnetic properties of sensing material or change of parameters of the magnetic circuit [1]. Magnetic sensors are more reliable and robust to dust and dirt, which is common problem in many applications. Magnetic sensors could have moving or stationary permanent magnet or electric circuit as magnetic source. They are contactless and have high possibility to be implemented in dirty and high temperature environment [2]. Contactless translational speed and position sensors are required for the pneumatic and hydraulic cylinders, flat type or tubular linear motors and actuators and generators for free piston engine and other industrial applications [3]-[4]. Available contactless magnetic sensors require non-smooth moving metallic part or permanent magnets for excitation. Another disadvantage of the existing solution is strong dependence on the distance. Speed probe based on eddy currents in metallic body moving in the field of permanent magnet was using Hall sensor, which has poor offset stability [5]. Using speed effects on the induced eddy currents of permanent magnet fields in the moving part was also studied in [6] for metal part defect detection. Speed sensor based on linear variable differential transformer (LVDT) operation with ferrite yoke is presented in [7], which was presented for flat type moving part. A speed sensor based on high sensitivity of an amorphous core with ring shape mounted on solid iron $\mathrm{E}$ shape core for magnetic field excitation was shown in [8], which has disadvantage of the necessity of using amorphous core with zero-magnetostriction and sharp rectangular $B-H$ curve.

Our solution for linear speed measurement is based on single coil and coil pair with DC and AC excitation. We present fast and precise analytical method to calculate coil inductance and induced voltages in the pick up coils taking into account eddy currents in the iron rod caused by alternating current and iron rod speed for an axisymmetric model. Finite element method (FEM) simulations and

\footnotetext{
Corresponding author: M. Mirzaei (e-mail: mirzameh@fel.cvut.cz).
}

experimental results are also presented.

\section{Model AND MATERIAl DATA}

Fig. 1 shows axisymmetric 3D model and 2D model with concentric stationary coils and moving iron rod. Three coils are implemented for magnetic field excitations and measurements for two different configurations. First configuration, model A has one excitation coil (number 2 in Fig. 1) and two antiserially connected pick up coils for measurement (numbers 1 and 3). Second configuration, model B has one pick up coil (number 2) and two excitation coils (numbers 1 and 3), which are antiserially connected. The speed of moving iron rod is in axial direction. Parameters $L, r_{\mathrm{i}}$, $r_{\mathrm{c}}, d, w$ and $h$ are axial length of iron rod, outer radius of iron rod, inner radius of coils, coils distance, coils thickness and coils height, respectively.

Solid iron is used for the moving iron rod. Initial relative magnetic permeability is only considered for magnetic analysis because of low magnetic fields, which is estimated to be about 77.5 [9]. Electrical conductivity of solid iron rod is $4.45 \mathrm{MS} / \mathrm{m}$ at room temperature.

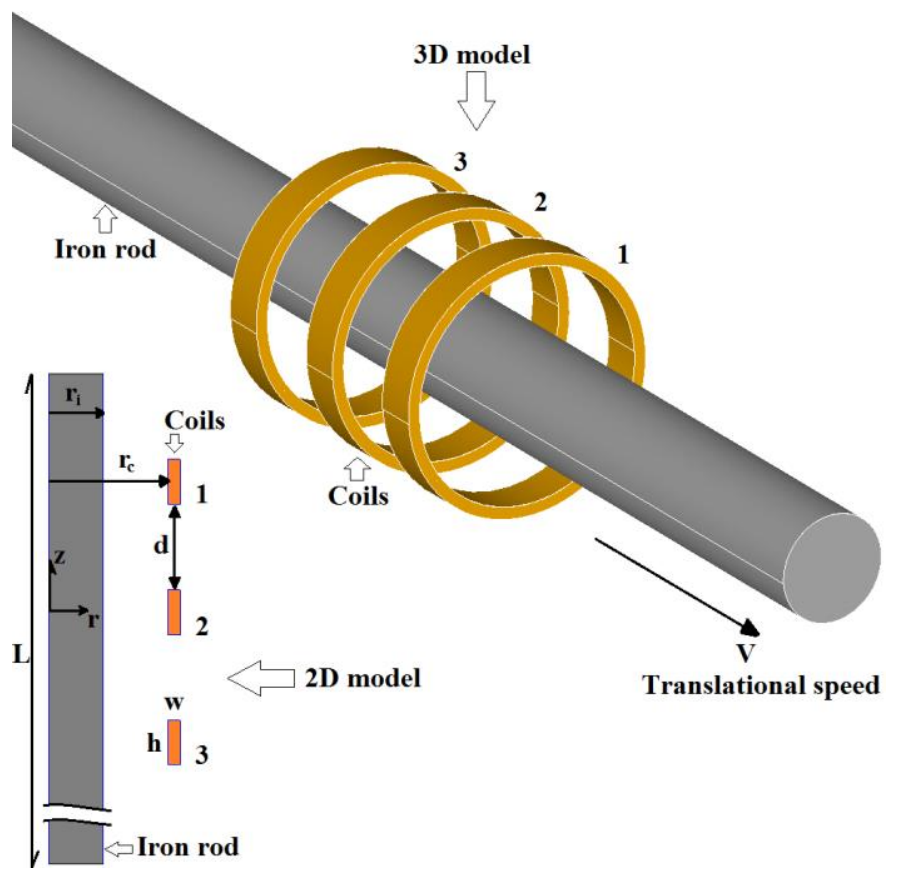


Fig. 1. The speed sensor coils and iron rod - 3D model and 2D model

\section{ANALYTICAL MODELING}

The Maxwell equations are used to compute speed sensor performance [10]. Final differential equations in cylindrical coordinate have only azimuthal-angle component of magnetic vector potential, $A_{\varphi}$ and current, $J_{\varphi}$ because of $2 \mathrm{D}$ axisymmetric configuration. Magnetic flux density and magnetic field strength have only radial and axial components, $B_{\mathrm{r}}, B_{\mathrm{z}}, H_{\mathrm{r}}$ and $H_{\mathrm{z}}$, respectively:

$$
\begin{aligned}
& {\left[\begin{array}{l}
B_{r} \\
B_{z}
\end{array}\right]=\left[\begin{array}{ll}
\mu & 0 \\
0 & \mu
\end{array}\right]\left[\begin{array}{l}
H_{r} \\
H_{z}
\end{array}\right], \frac{\partial H_{r}}{\partial z}-\frac{\partial H_{z}}{\partial r}=J_{\varphi}, \frac{1}{r} \frac{\partial\left(r H_{r}\right)}{\partial r}+\frac{\partial H_{z}}{\partial z}=0} \\
& {\left[\begin{array}{l}
-\frac{\partial J_{\varphi}}{\partial z} \\
\frac{1}{r} \frac{\partial\left(r J_{\varphi}\right)}{\partial r}
\end{array}\right]=\left[\begin{array}{l}
-\sigma \frac{d B_{r}}{d t} \\
-\sigma \frac{d B_{z}}{d t}
\end{array}\right],\left[\begin{array}{l}
B_{r} \\
B_{z}
\end{array}\right]=\left[\begin{array}{l}
-\frac{\partial A_{\varphi}}{\partial z} \\
\frac{1}{r} \frac{\partial\left(r A_{\varphi}\right)}{\partial r}
\end{array}\right]}
\end{aligned}
$$

where, $\mu$ and $\sigma$ are magnetic permeability and electrical conductivity, respectively.

The induced eddy current in the iron rod with electrical conductivity, $\sigma_{\mathrm{i}}$ due to the iron rod speed, $V$ must be considered in the equations:

$$
J_{\varphi}=-\sigma_{i} \frac{d A_{\varphi}}{d t}=-\sigma_{i}\left(\frac{\partial A_{\varphi}}{\partial t}+\frac{d z}{d t} \cdot \frac{\partial A_{\varphi}}{\partial z}\right)=-\sigma_{i}\left(\frac{\partial A_{\varphi}}{\partial t}+V \cdot \frac{\partial A_{\varphi}}{\partial z}\right)
$$

The computational model is divided to four parts. Part 1, 2, 3 and 4 are iron rod region, air region between iron rod and coils, coils region and air region beyond coils, respectively.

$$
\begin{aligned}
& \frac{1}{r} \frac{\partial}{\partial r}\left(r \frac{\partial A_{\varphi, 1}}{\partial r}\right)+\frac{\partial^{2} A_{\varphi, 1}}{\partial z^{2}}-\frac{A_{\varphi, 1}}{r^{2}}=\sigma_{i} \cdot \mu_{1} \cdot\left(\frac{\partial A_{\varphi, 1}}{\partial t}+V \cdot \frac{\partial A_{\varphi, 1}}{\partial z}\right) \\
& \frac{1}{r} \frac{\partial}{\partial r}\left(r \frac{\partial A_{\varphi, 3}}{\partial r}\right)+\frac{\partial^{2} A_{\varphi, 3}}{\partial z^{2}}-\frac{A_{\varphi, 3}}{r^{2}}=-\mu_{3} \cdot J_{s} \\
& \frac{1}{r} \frac{\partial}{\partial r}\left(r \frac{\partial A_{\varphi, 2,4}}{\partial r}\right)+\frac{\partial^{2} A_{\varphi, 2,4}}{\partial z^{2}}-\frac{A_{\varphi, 2,4}}{r^{2}}=0
\end{aligned}
$$

where, $J_{\mathrm{s}}$ is the current density in the coils.

The method of separation of variables (method of Fourier) is used to solve (3), (4) and (5) [10].

They are assumed that magnetic fields are changing sinusoidal versus time and periodically in $\mathrm{z}$-direction with period length $2 L$. Therefore derivations could be replaced as following:

$$
A_{\varphi}=R(r) \cdot Z(z) \cdot T(t)
$$

$Z(z) \propto \exp (-j m \cdot z), m=\frac{n \pi}{L}$

$T(t) \propto \exp (j \omega \cdot t)$

$\frac{\partial A_{\varphi, 1}}{\partial t}=j \omega \cdot A_{\varphi, 1}$

$\frac{\partial A_{\varphi, 1}}{\partial z}=-j m \cdot A_{\varphi, 1}, \frac{\partial^{2} A_{\varphi, 1}}{\partial z^{2}}=-m^{2} \cdot A_{\varphi, 1}$

where, $\omega$ is angular frequency in $\mathrm{rad} / \mathrm{s}$.

The solutions versus radius, $r$ are as follows:

$$
\begin{aligned}
& A_{\varphi, 1}=\sum_{n= \pm 1, \pm 3, \ldots}\left(C_{11} \cdot J_{1}(\gamma \cdot r)+C_{12} \cdot Y_{1}(\gamma \cdot r)\right) \\
& \gamma=\sqrt{-m^{2}-j \mu_{1} \sigma_{\mathrm{i}}(\omega-m \cdot V)} \\
& A_{\varphi, 2}=\sum_{n= \pm 1, \pm 3, \ldots}\left(C_{21} \cdot I_{1}(m \cdot r)+C_{22} \cdot K_{1}(m \cdot r)\right) \\
& A_{\varphi, 3}=\sum_{n= \pm 1, \pm 3, \ldots}\left(C_{31} \cdot I_{1}(m \cdot r)+C_{32} \cdot K_{1}(m \cdot r)-\frac{\mu_{3} J_{\mathrm{m}} \pi}{2 m^{2}} \cdot L_{1}(m \cdot r)\right) \\
& A_{\varphi, 4}=\sum_{n= \pm 1, \pm 3, \ldots}\left(C_{41} \cdot I_{1}(m \cdot r)+C_{42} \cdot K_{1}(m \cdot r)\right)
\end{aligned}
$$

where, $J_{1}, Y_{1}, I_{1}$ and $K_{1}$ are Bessel functions and $L_{1}$ is Struve function. $C_{11}, C_{12}, C_{21}, C_{22}, C_{31}, C_{32}, C_{41}$ and $C_{42}$ are constants, which are obtained by the following boundary conditions:

$A_{\varphi, 1}(r=0)=0$

$A_{\varphi, 1}\left(r=r_{\mathrm{i}}\right)=A_{\varphi, 2}\left(r=r_{\mathrm{i}}\right), H_{z, 1}\left(r=r_{\mathrm{i}}\right)=H_{z, 2}\left(r=r_{\mathrm{i}}\right)$

$A_{\varphi, 2}\left(r=r_{\mathrm{c}}\right)=A_{\varphi, 3}\left(r=r_{\mathrm{c}}\right), H_{z, 2}\left(r=r_{\mathrm{c}}\right)=H_{z, 3}\left(r=r_{\mathrm{c}}\right)$

$A_{\varphi, 3}\left(r=r_{\mathrm{c}}+w\right)=A_{\varphi, 4}\left(r=r_{\mathrm{c}}+w\right)$,

$H_{z, 3}\left(r=r_{\mathrm{c}}+w\right)=H_{z, 4}\left(r=r_{\mathrm{c}}+w\right)$

$A_{\varphi, 3}(r=\infty)=0$

Parameter, $J_{\mathrm{m}}$ in (8) for coils region for single coil excitation and two antiserially connected coils could be calculated as follows, respectively:

$J_{\mathrm{m}}=\frac{2}{n \pi} \cdot \sin \left(m \cdot \frac{h}{2}\right) \cdot J_{\mathrm{s}}$

$J_{\mathrm{m}}=-\frac{2}{j n \pi} \cdot\left(\cos \left(m \cdot\left(d+3 \cdot \frac{h}{2}\right)\right)-\cos \left(m \cdot\left(d+\frac{h}{2}\right)\right)\right) \cdot J_{\mathrm{s}}$

$J_{\mathrm{s}}=\frac{N I}{h \cdot w}$

where, $N$ and $I$ are the number of turns per coil and current, respectively. Struve function, $L_{1}$ is complicated for calculation and shows instability. An approximation is used to replace Struve function, $L_{1}$ with simpler one. It is assumed that current density changes directly proportional or inversely proportional with radius and it is not constant in the coil cross section. Therefore solutions of (4) for magnetic vector potentials, $A_{\varphi, 3}$ are as following for linear current change and inversely current change with radius, respectively: 


$$
\begin{aligned}
& \frac{1}{r} \frac{\partial}{\partial r}\left(r \frac{\partial A_{\varphi, 3}}{\partial r}\right)+\frac{\partial^{2} A_{\varphi, 3}}{\partial z^{2}}-\frac{A_{\varphi, 3}}{r^{2}}=-\mu_{3} J_{s} r, J_{s}=\frac{2 N I}{h\left(w^{2}+2 w r_{\mathrm{c}}\right)} \\
& A_{\varphi, 3}=\sum_{n= \pm 1, \pm 3, \ldots}\left(C_{31} \cdot I_{1}(m \cdot r)+C_{32} \cdot K_{1}(m \cdot r)+\frac{\mu_{3} J_{\mathrm{m}}}{m^{2}} \cdot r\right) \\
& \frac{1}{r} \frac{\partial}{\partial r}\left(r \frac{\partial A_{\varphi, 3}}{\partial r}\right)+\frac{\partial^{2} A_{\varphi, 3}}{\partial z^{2}}-\frac{A_{\varphi, 3}}{r^{2}}=-\mu_{3} \frac{J_{s}}{r}, J_{s}=\frac{N I}{h \cdot \ln \left(1+\frac{w}{r_{\mathrm{c}}}\right)} \\
& A_{\varphi, 3}=\sum_{n= \pm 1, \pm 3, \ldots}\left(C_{31} \cdot I_{1}(m \cdot r)+C_{32} \cdot K_{1}(m \cdot r)+\frac{\mu_{3} J_{\mathrm{m}}}{m^{2} \cdot r}\right)
\end{aligned}
$$

\section{EXPERIMENTAL AND SIMULATION RESULTS}

Fig. 2 and Table I show experimental setup for eddy current linear speed sensor and parameters. The axial length of iron rod is considered large enough to decrease end effects caused by finite length of iron rod. The number of turns per coil, $N$ is 47. Fig. 3 and Fig. 4 show magnetic flux distribution in models A and B at DC current and iron rod speed $0 \mathrm{~m} / \mathrm{s}$ and $5 \mathrm{~m} / \mathrm{s}$, which calculated by analytical method. The effect of speed on the flux distribution could be more distinguished in the iron rod region. The induced eddy current due to the iron rod speed causes unsymmetrical magnetic flux distribution. Self inductance, $L_{\mathrm{S}}$ and mutual inductance, $L_{\mathrm{M}}$ could be calculated as follows [11]:

$$
L_{\mathrm{S}}, L_{\mathrm{M}}=\frac{\Psi}{I}=\frac{N \int A_{\varphi} \cdot d V}{I \cdot h w}
$$

where, $\Psi$ is the total average flux linkage or mutual flux linkage over coils cross section area. The integration is applied on each coil volume. Table II shows experimental and analytical results for self and mutual inductances between adjacent coils at zero speed, which presents appropriate precision for the analytical method. The self inductance has only inductive component in Table II and mutual inductance has inductive and resistive component, where the resistive component is caused by induced eddy currents in the iron rod. Eddy currents are only generated in the regions where the excitation field has radial component. Fig. 5 shows analytical and FEM results for the coils inductances and induced voltages of models $\mathrm{A}$ and $\mathrm{B}$.

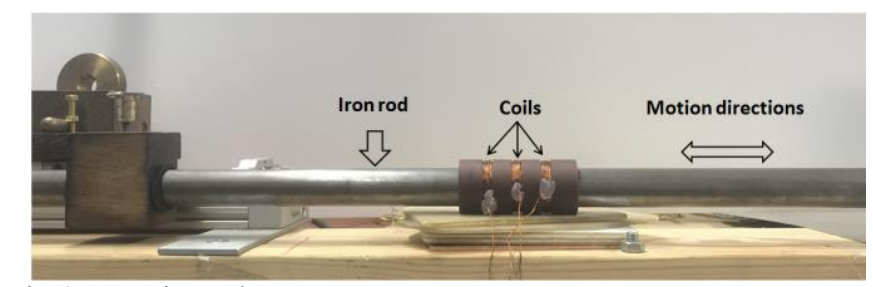

Fig. 2. Experimental set up
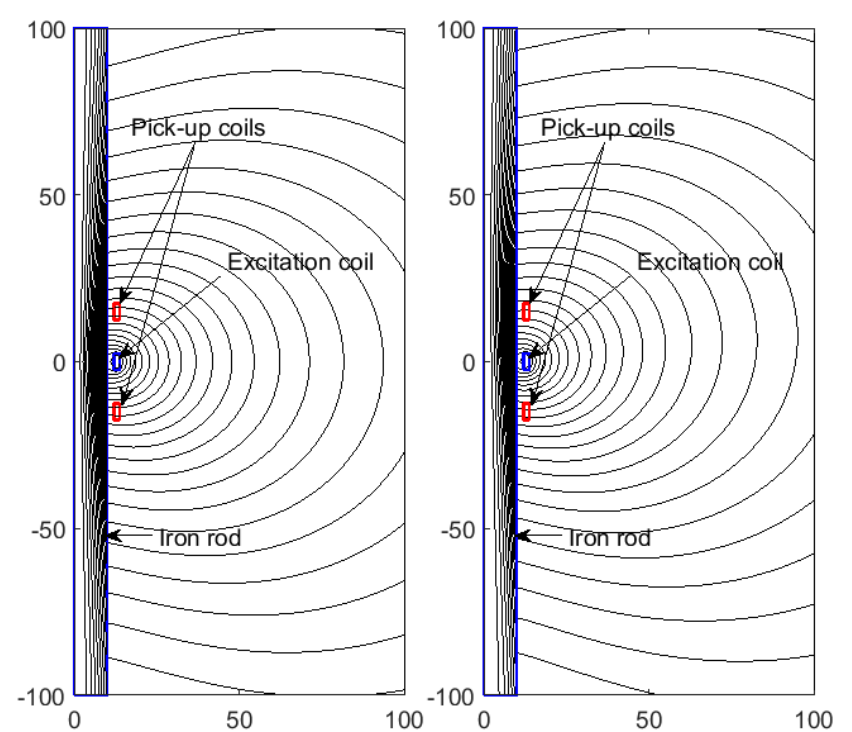

Fig. 3. Magnetic flux distribution in model $A$ at DC current $(0 \mathrm{~m} / \mathrm{s}$ (left) and $+5 \mathrm{~m} / \mathrm{s}$ (right))
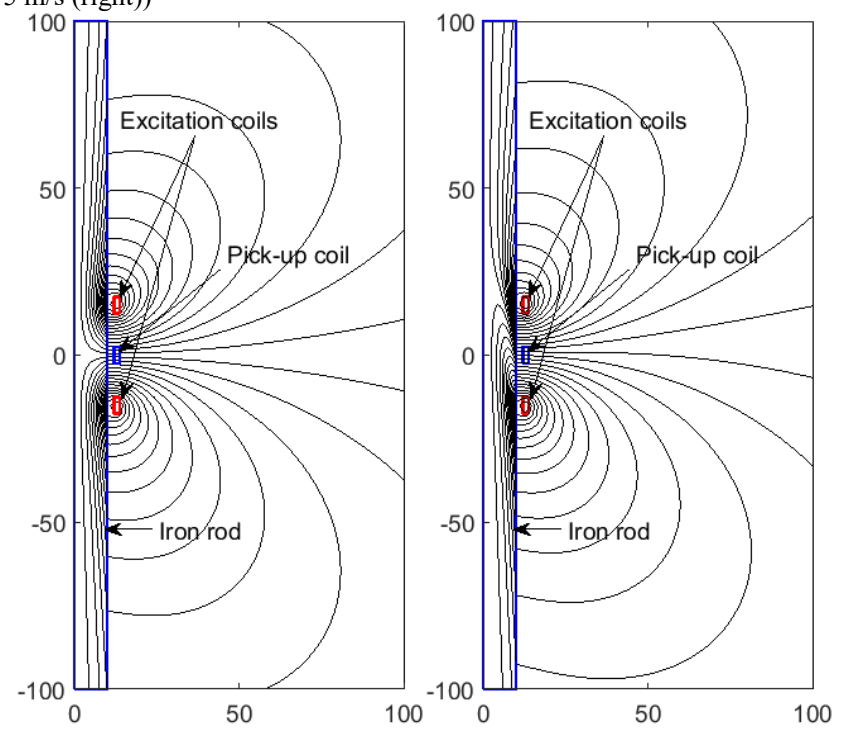

Fig. 4. Magnetic flux distribution in model B at DC current -two excitation coils are connected antiserially ( $0 \mathrm{~m} / \mathrm{s}$ (left) and $+5 \mathrm{~m} / \mathrm{s}$ (right))

TABLE I

PARAMETERS OF SPEED SENSOR

\begin{tabular}{llllllll}
\hline \hline Parameters & $w$ & $h$ & $r_{\mathrm{c}}$ & $d$ & $r_{\mathrm{i}}$ & $L$ \\
\hline unit (mm) & 1.8 & 5.0 & 12.0 & 10.0 & 10.0 & 700.0 \\
\hline
\end{tabular}

TABLE II

COMPARISON BETWEEN EXPERIMENTAL AND ANALYTICAL RESULTS MODEL A (EXCITATION CURRENT AMPLITUDE $=0.165 \mathrm{~A})$

\begin{tabular}{lcc}
\hline \hline & Experimental & Analytical \\
\hline$L_{\mathrm{S} \text {-air }}$ & $85.0(\mu \mathrm{H})$ & $79.8(\mu \mathrm{H})$ \\
$L_{\mathrm{M} \text {-air }}$ & $12.4(\mu \mathrm{H})$ & $11.5(\mu \mathrm{H})$ \\
$L_{\mathrm{S} \text {-iron, } 100 \mathrm{~Hz}}$ & $312.0(\mu \mathrm{H})$ & $291.1(\mu \mathrm{H})$ \\
$L_{\mathrm{M} \text {-iron, } 100 \mathrm{~Hz}}$ & $209.3(\mu \mathrm{H})$ & $181.8(\mu \mathrm{H})$ \\
$L_{\mathrm{S} \text {-iron, } 1000 \mathrm{~Hz}}$ & $195.0(\mu \mathrm{H})$ & $185.0(\mu \mathrm{H})$ \\
$L_{\mathrm{M} \text {-iron, } 1000 \mathrm{~Hz}}$ & $98.6(\mu \mathrm{H})$ & $85.4(\mu \mathrm{H})$ \\
\hline
\end{tabular}



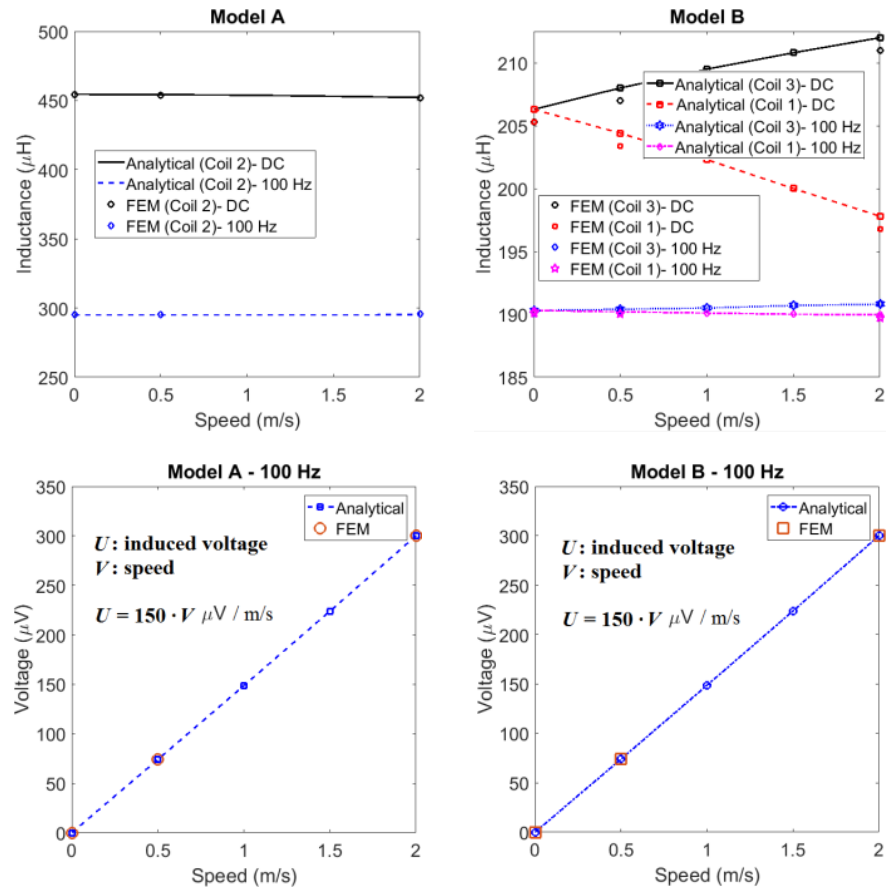

Fig. 5. Coils inductances (up) and induced voltages (bottom) - induced voltage in model $\mathrm{A}$ is the difference voltage between pick up coils 1 and 3

The inductances changes is negligible with speed in model $\mathrm{A}$ at $\mathrm{DC}$ current and $100 \mathrm{~Hz} \mathrm{AC}$ current. The variations of inductances are more obvious in model $\mathrm{B}$, which could be used as speed meter. The induced voltages amplitude changes linearly with speed in Fig. 5. The induced voltage amplitude speed ratio is about $150 \mu \mathrm{V} /(\mathrm{m} / \mathrm{s})$. The experimental and analytically calculated results for induced voltage of models $\mathrm{A}$ and $\mathrm{B}$ versus time at different speeds are shown in Fig. 6 and Fig. 7. The experimental voltage results are recorded by oscilloscope for a time range. The analytical induced voltages coincide well with experimental results, which show accuracy of the proposed analytical method. The acceleration effects are neglected in the analytical modeling and only pure inductive effects are considered. The sign of speed could not been observed from amplitude of induced voltage but it could be computed by relative phase angle to excitation current.

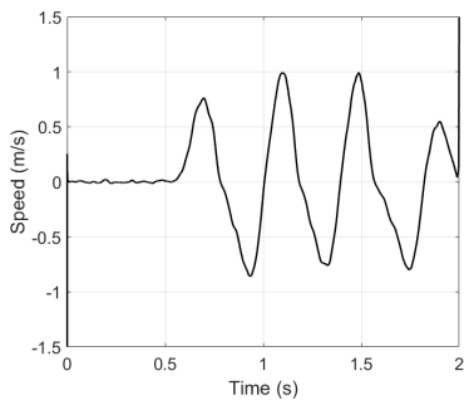

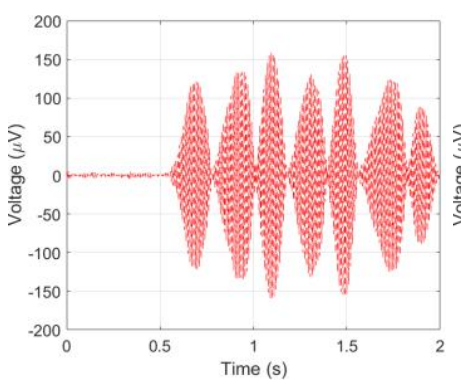

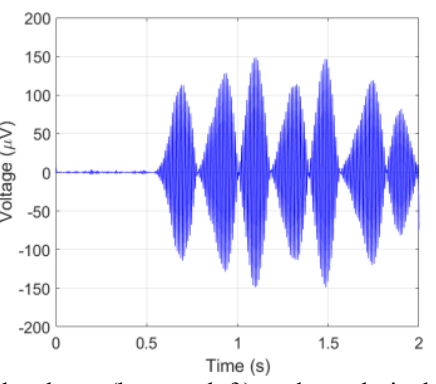

Fig. 6. Measured speed (up), measured voltage (bottom-left) and analytical calculated voltage (bottom-right) - model A with current amplitude $165 \mathrm{~mA}$
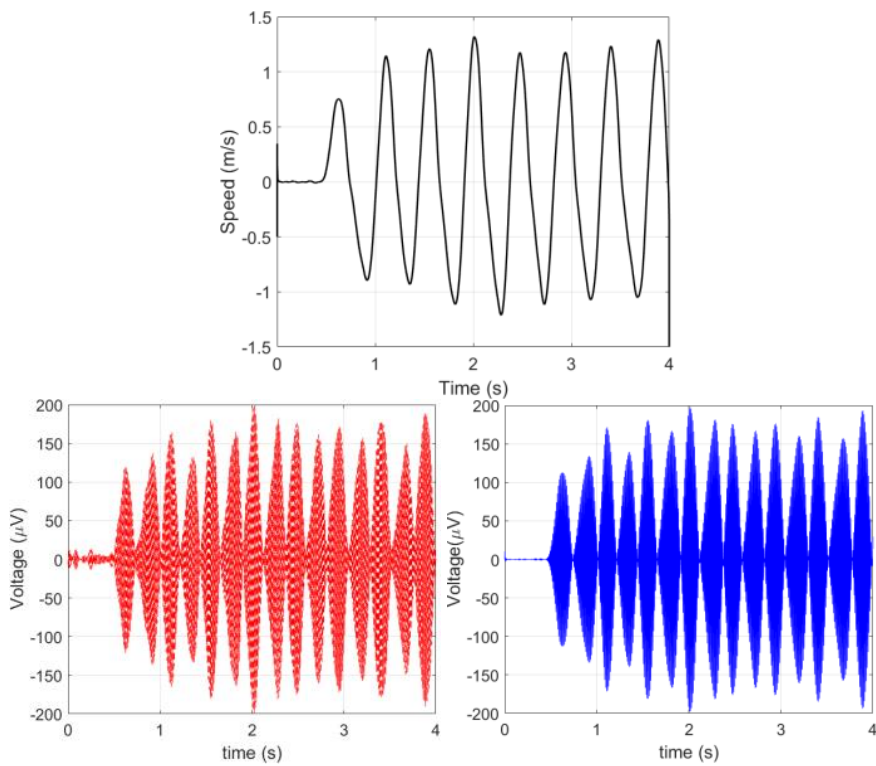

Fig. 7. Measured speed (up), measured voltage (bottom-left) and analytical calculated voltage (bottom-right) - model B with current amplitude $165 \mathrm{~mA}$

The comparison between estimated speed and measured speed (Fig. 6 and Fig. 7) is shown in Fig. 8, which presents appropriate accuracy. The induced voltage amplitude - speed ratio, $150 \mu \mathrm{V} /(\mathrm{m} / \mathrm{s})$ is used to convert induced voltages to the speed. The estimated speed results could be obtained using voltage peaks or voltage RMS or rectified DC value in each half period.
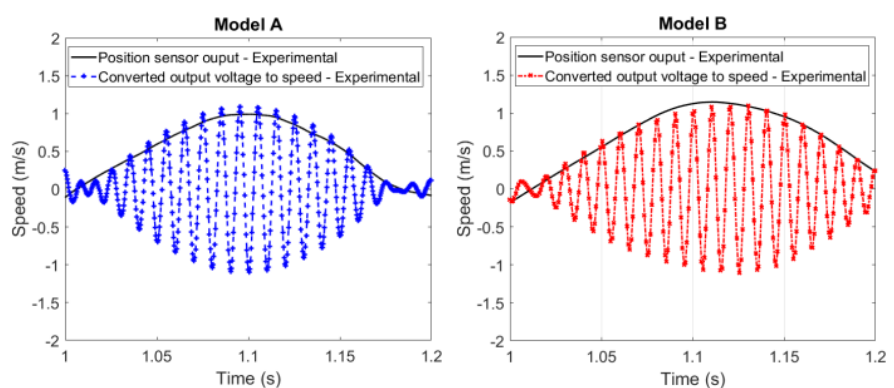

Fig. 8. Comparison between estimated speed and measured speed

\section{CONCLUSIONS}

A translational speed sensor for an axisymmetric moving part was presented. Analytical model results coincide well with FEM results and experimental results. Analytical model could be used for speed sensor optimization. Inductances of excitation coils linearly change with speed at DC currents and low frequencies in model $\mathrm{B}$, which could be used as speed 
meter. Pick-up coils voltage versus speed is a linear curve. The presented speed sensor could be used for linear machines and pneumatic and hydraulic cylinders.

\section{REFERENCES}

[1] P. Ripka, Magnetic Sensors and Magnetometers, Artech House, Jan 1, 2001 - Technology \& Engineering - 494 pages

[2] S. Tumanski, Handbook of Magnetic Measurements, June 23, 2011 by CRC Press Reference - 404 Pages

[3] P. Zheng, C. Tong, G. Chen, R. Liu, Y. Sui, W. Shi, and S. Cheng, "Research on the magnetic characteristic of a novel transverse-flux PM linear machine used for free-piston energy converter," IEEE Transactions on Magnetics, Year: 2011, Volume: 47, Issue: 5, Pages: $1082-1085$

[4] Y. Sui, P. Zheng, B. Yu, L. Cheng, and Z. Liu, "Research on a tubular yokeless linear PM machine," IEEE Transactions on Magnetics, Year: 2015, Volume: 51, Issue: 11

[5] E. Cardelli, A. Faba, and F. Tissi, "Contact-Less Speed Probe Based on Eddy Currents," IEEE Transactions on Magnetics, Year: 2013, Volume: 49, Issue: 7, Pages: 3897 - 3900

[6] T. J. Rocha, H. G. Ramos, A. L. Ribeiro, and D. J. Pasadas, "Evaluation of subsurface defects using diffusion of motion-induced eddy currents," IEEE Transactions on Instrumentation and Measurement, Year: 2016, Volume: 65, Issue: 5, Pages: 1182 - 1187

[7] A. Tuysuz, M. Flank1, J. W. Kolar, and A. Mutze, "Eddy-current-based contactless speed sensing of conductive surfaces," 2016 IEEE 2nd Annual Southern Power Electronics Conference (SPEC), Year: 2016, Pages: 1 - 6

[8] T. Sonoda, R. Ueda, K. Fujitani, T. Irisa, and S. Tatata, "DC magnetic field type eddy current speed sensor detecting cross magnetization field with amorphous core," IEEE Transactions on Magnetics, Year: 1985, Volume: 21, Issue: 5, Pages: 1732 - 1734

[9] P. Ripka, A. Chirtsov, and M. Mirzaei, "Inductance position sensor for pneumatic cylinder", AIP Advances 8, 048001 (2018)

[10] M. N. O. Sadiku, Numerical Techniques in Electromagnetics, Second Edition, July 12, 2000 by CRC Press, Textbook - 760 Pages

[11] K. Davey, "Analytic analysis of single- and three-phase induction motors," IEEE Transactions on Magnetics, Year: 1998, Volume: 34, Issue: 5 